\title{
Modelling the South African fruit export infrastructure: A case study
}

\author{
FG Ortmann* $\quad$ JH van Vuuren ${ }^{\dagger} \quad$ FE van Dyk ${ }^{\ddagger}$ \\ Received: 2 January 2006; Revised: 18 April 2006; Accepted: 21 April 2006
}

\begin{abstract}
A description is provided of work performed as part of the fruit logistics infrastructure project commissioned by the South African Deciduous Fruit Producers' Trust and coordinated by the South African Council for Scientific and Industrial Research, as described in VAN DYK FE \& Maspero E, 2004, An analysis of the South African fruit logistics infrastructure, ORiON, 20(1), pp. 55-72. After a brief introduction to the problem, two models (a single-commodity graph theoretic model and a multi-commodity mathematical programming model) are derived for determining the maximal weekly flow or throughput of fresh fruit through the South African national export infrastructure. These models are solved for two extreme seasonal export scenarios and the solutions show that no export infrastructure expansion is required in the near future - observed bottlenecks are not fundamental to the infrastructure and its capacities, but are rather due to sub-optimal management and utilisation of the existing infrastructure.
\end{abstract}

Key words: Maximum flow; single/multi-commodity flow; fresh fruit export infrastructure.

\section{Introduction}

This paper contains a description of infrastructure capacity analyses conducted during the third phase of the national fruit logistics infrastructure study (Fruitlog), commissioned by the South African Deciduous Fruit Producers' Trust, as described in [20]. The specific task of the authors within this larger project was to construct "a maximum flow optimisation model for the [South African] infrastructure network ... to determine the maximum volume of [fresh fruit] produce that may be handled by certain sectors of the network." This paper is organised as follows. After describing a number of modelling assumptions in $\S 2$, a single-commodity graph theoretic model is derived in $\S 2.1$ for determining the maximum weekly flow of fresh fruit exported through the national transportation infrastructure. Due

\footnotetext{
${ }^{*}$ Corresponding author: Department of Mathematical Sciences, University of Stellenbosch, Private Bag X1, Matieland, South Africa, 7602, email: ortmann@dip.sun.ac.za

${ }^{\dagger}$ Department of Mathematical Sciences, University of Stellenbosch, Private Bag X1, Matieland, South Africa, 7602

${ }^{\ddagger}$ CSIR — Transportek, PO Box 320, Stellenbosch, 7599
} 
to certain shortcomings of this model, an improved multi-commodity mathematical programming model is derived in $\S 2.2$. This is followed, in $\S 3$, by a discussion of the process of gathering the necessary data in order to solve the mathematical models of $\S 2$. The models are then solved in $\S 4$ for two extreme seasonal scenarios designed by the authors (based on past export data) in order to ascertain the current level of utilisation of the export infrastructure and to answer the question as to whether export infrastructure expansion is required. Finally some conclusions and recommendations are made in $\S 5$.

\section{Development of models}

The South African national fruit export infrastructure may be modelled as a directed network. Each node in the network represents a physical component of the infrastructure or a possible decision point. Examples of physical infrastructure components include pack houses, cold stores and terminals (see Figure 1 for a simplied schematic representation of the export process). Decision nodes represent points during the export process where decisions have to be made, such as transportation of fruit by road or rail, shipments of fruit via conventional vessel or container vessel, in break-bulk or containerised packing, refrigerated or unrefrigerated, etc. Arcs link the nodes in the network and represent possible avenues of flow of commodities (fruit groupings in this case). These arcs have various attributes associated with them, such as minimum flow requirements, maximum flow restrictions and restrictions on commodity types allowed on the arcs.

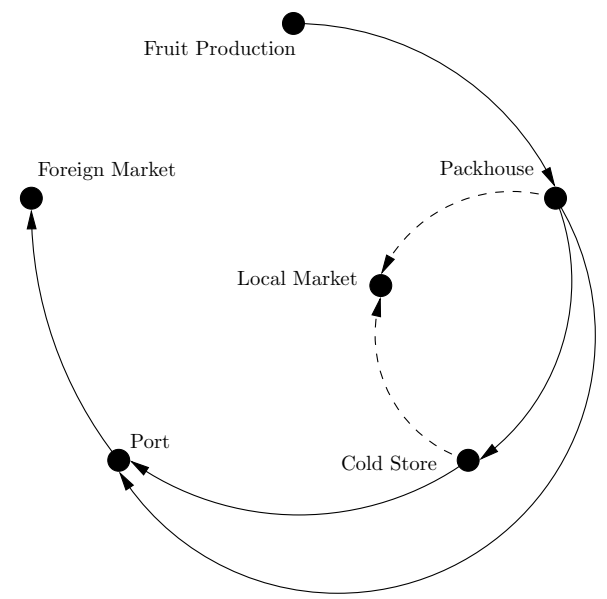

Figure 1: Simplified schematic of fruit flow in the South African export supply chain.

The 850 pack houses and 286 cold stores countrywide where fresh fruit is packed and cooled (excluding those at the ports) were grouped into 31 pack house and cold store regions (shown in Figure 2). These production regions are generally the same regions as those specified by producer associations such as Citrus South Africa (CSA), the Deciduous Fruit Producers' Trust (DFPT), the South African Avocado Growers' Association (SAAGA), the South African Mango Growers' Association (SAMGA) and the South African Litchi Growers' Association (SALGA). This choice was made to minimise confusion when interested parties interpret model solutions, and in a bid to model fruit production information 
as accurately as possible. Some of these regions only represent a small number of farms, pack houses and cold stores in or near a single town (such as the De Doorns region, which contains only the infrastructure in and around the small Western Cape town of De Doorns), while other regions encompass a large number of towns (such as the Boland region, which includes all infrastructure in and near Somerset West, Stellenbosch, Simondium, Paarl, Wellington, Windmeul, Paardeberg, Groendal and Groot Drakenstein). A significantly simplified extract of the resulting export infrastructure network (omitting the majority of production regions for the sake of clarity) is shown in Figure 3.

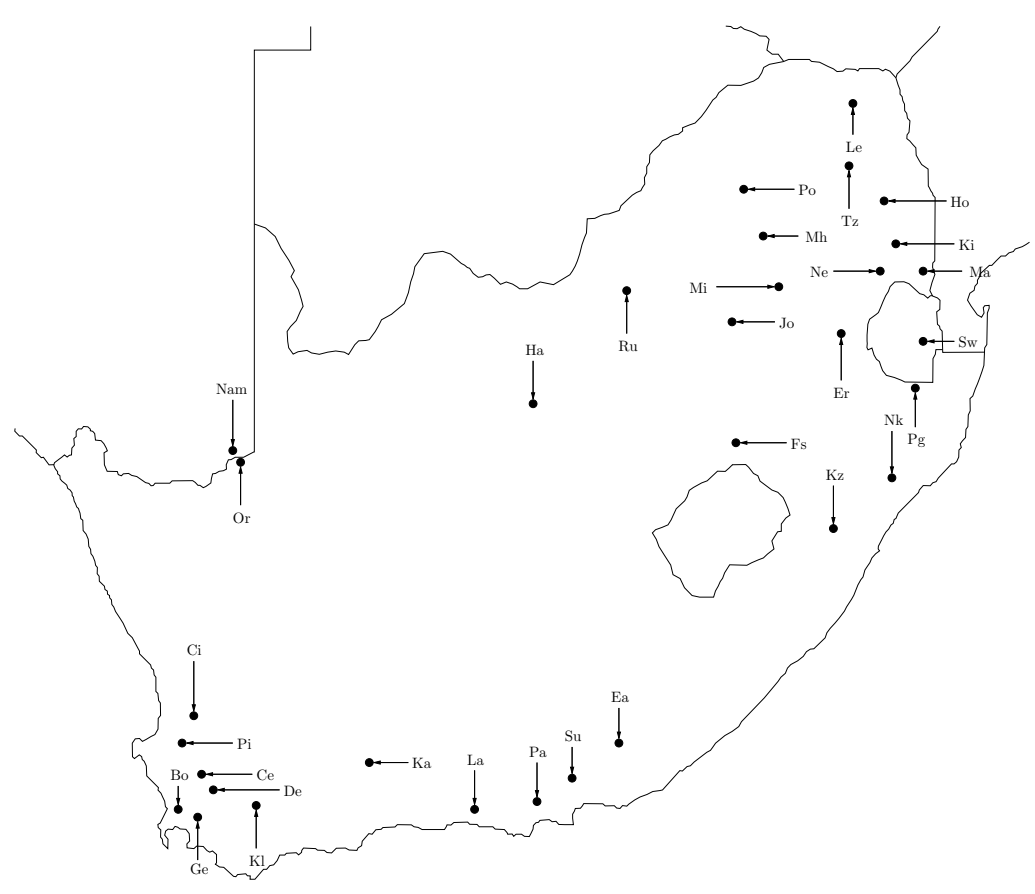

Figure 2: $\quad$ The 31 fruit production regions in South Africa. Table 2 contains the place names represented by 2 letter abbreviations in the figure. Pack house and cold store data for Namibia (Nam) and Swaziland $\left(\mathrm{SW}_{\mathrm{w}}\right.$ ) could not be found and are thus not considered in our model, even though fruit grown in those regions are generally exported via South Africa.

Four central modelling assumptions were made throughout. The first assumption is that all export fruit takes preference over fruit sold on the local market. Under this assumption we consider export volumes only and disregard the remaining fruit (sold locally, juiced or processed in other ways). Past export volumes may be found with some degree of accuracy, as all such fruit pass cold store and port inspections, records of which are kept by the Perishable Products Export Control Board (PPECB) - the national inspection body and distributed to the various producer organisations. To determine the volumes of fruit sold or processed locally would be near impossible, as all producers have their individual methods of profiting from sub-export quality fruit. This assumption does not affect the realism of the model significantly, since export fruit would generally take preference over local fruit in situations of congestion [14]. 


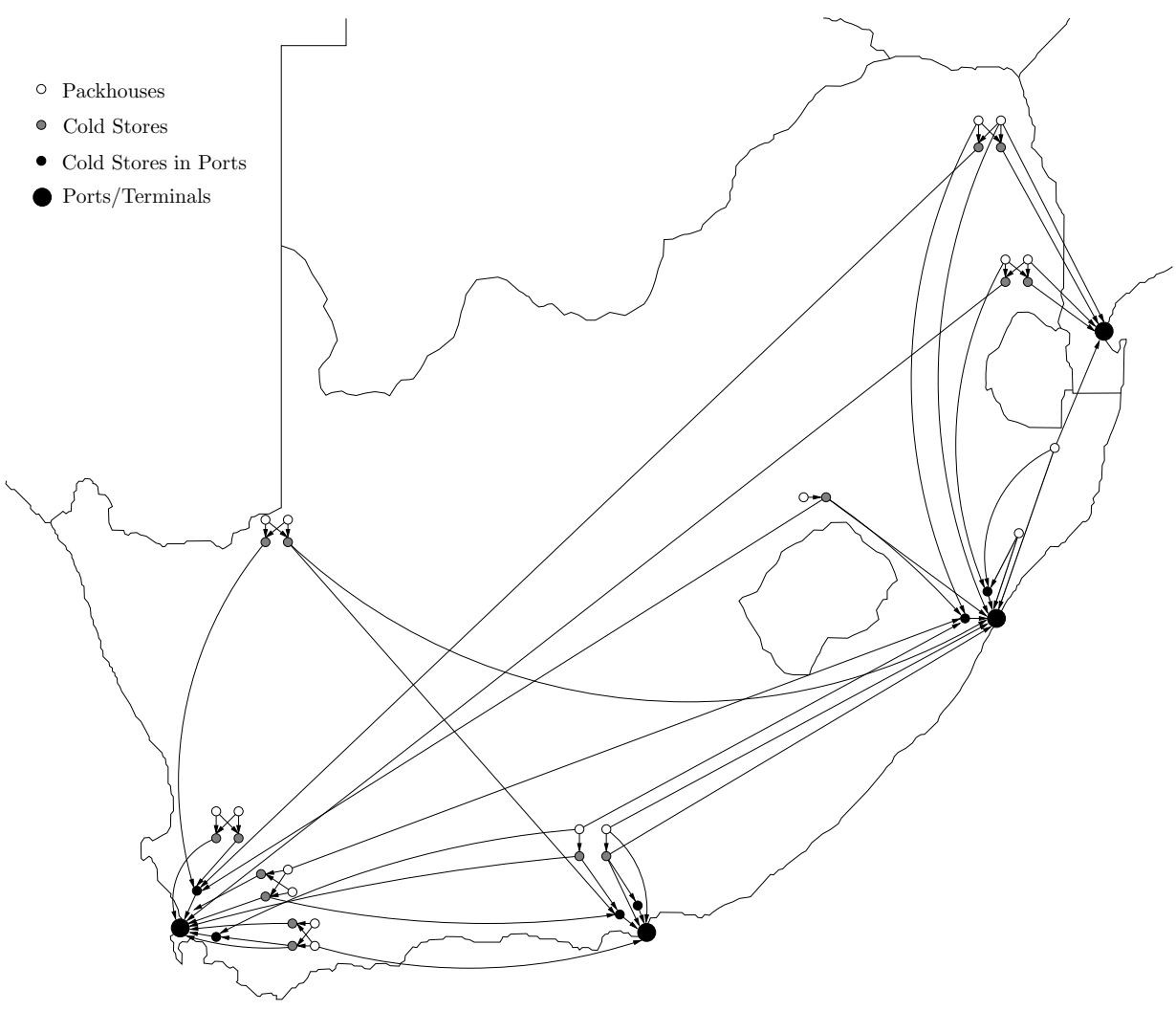

Figure 3: Simplified schematic representation of the South African fruit export infrastructure network (the majority of fruit regions have been omitted from the figure for the sake of clarity).

Ignoring the possibility of exporting fruit by air freight is the second assumption. This assumption forces all fruit to be exported via the ports. The effect of this assumption on the realism of the model is negligible, as air freight is used on very rare occasions only. One such occasion is, for example, when producers in the Orange River region harvest table grapes. If harvesting in this region occurs early enough to allow shipment to Europe so that the fruit arrives at the foreign market before Christmas, then these grapes are exported via sea freight. However, if harvesting occurs too late, then the grapes are flown out to Europe so as to arrive there before Christmas, when table grape prices are high enough to warrant the significantly higher air freight transportation costs.

Table grapes are somewhat different to the other fruit types in the sense that it is generally transported from farms to the farmers' own pack houses to be packed (as opposed to the other fruit groupings, for which pack houses are usually operated by independent companies or co-operatives). However, we assumed in our models that grapes are, in fact, transported directly from the farms to cold stores. This third assumption significantly decreases the number of pack houses for which data had to be gathered, as each table grape farmer generally has a pack house on his own farm [10]. The assumption does not have a large impact on the realism of the model, as each farmer generally ensures that his pack house has enough capacity to pack all fruit harvested on his farm. Otherwise, if a farm's pack house is too small, a farmer may come to an agreement with a nearby farmer 
(who has spare capacity in his pack house) to pack excess fruit.

The final assumption is that one may group the pack houses together in each of the 31 regions into a number of super-pack house nodes in which the same groupings of fruits are packed, and similarly for the cold stores. This significantly reduces the number of nodes required to represent the infrastructure network, and it would only be possible to relax this assumption if one were to know to which cold stores each individual pack house transported its fruit. However, the gathering of such detailed data would be extremely time-consuming, assuming that the managers of pack houses would be willing to part with such sensitive information. Even though this information is not known, one could still model each pack house individually, but as all pack houses in a region are adjacent in the infrastructure network to those cold stores in the region that cool the fruit groups packed by the pack house in question, it does not make a difference whether one groups such pack houses and cold stores together, if they pack or cool the same fruit groups. Thus the grouping together of infrastructure components does not influence the accuracy of the model if one does not know to which cold stores the individual pack houses send their fruit.

In the remainder of this section a single-commodity ${ }^{1}$ graph theoretic approach and a multicommodity mathematical programming approach is adopted towards modelling maximum weekly export throughput through the existing national infrastructure.

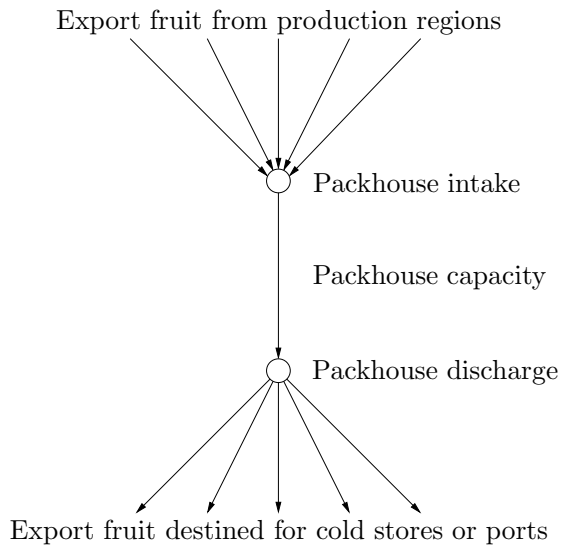

(a) A super-pack house

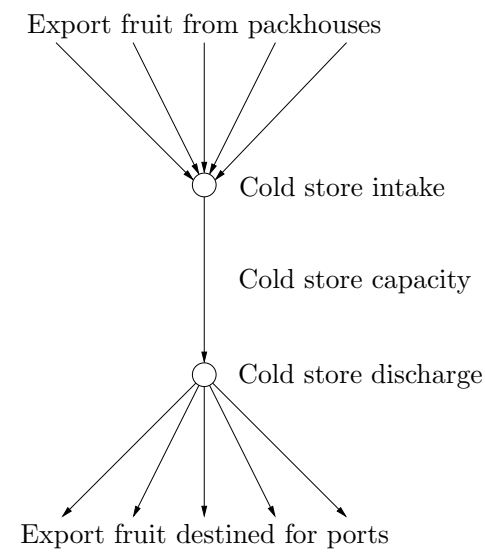

(b) A super-cold store

Figure 4: A section of the graph model illustrating the technique used to limit fruit flow to the capacities of super-pack houses and super-cold stores in the fruit export infrastructure. The same technique was used for port infrastructure such as cold stores and terminals.

\subsection{Single-commodity graph theoretic model}

The single-commodity graph model has a form similar to the network shown in Figure 3 , but includes all 31 regions. Fruit flow originates at nodes representing the production

\footnotetext{
${ }^{1}$ From here on the term "single-commodity" will be used in the sense that all fruit types are grouped together and treated as a single "commodity;" the term does not mean that only one fruit type is considered. The term "multi-commodity" will be used to denote the scenario where flows of different fruit groups are treated separately from a modelling point of view.
} 
regions for each fruit group. Pack houses within a region were grouped together to form super-pack houses depending on the fruit types that had been packed by them in the past according to a list of pack house and cold store data provided by the PPECB. Arcs join the production regions to nodes representing the intake points of super-pack houses. These arcs have very high fruit flow capacities associated with them, representing the fact that there is no significant restriction on the volume of fruit that may pass along the routes (typically roads) from production regions to super-pack houses. Flow constraints on the super-pack house nodes, representing accumulated maximum packing rates, were incorporated by adding artificial arcs between intake and discharge points at the superpack houses (see Figure 4(a)). This was done because standard maximum flow algorithms assume that flows are constrained on the arcs of a network only, and not on nodes. The same procedure was followed for the cold stores (see Figure 4(b)).

Furthermore, arcs lead from the discharge points of super-pack houses to the intake points at super-cold stores that cool at least one of those fruits packed by the corresponding super-pack houses. As hard citrus fruit may be transported to the ports at ambient temperatures, certain arcs originating from nodes representing discharge points of superpack houses packing hard citrus (and perhaps other fruits) lead to nodes representing intake points of cold stores in or near ports, or to terminals where break-bulk fruit is loaded onto vessels, via decision nodes representing unrefrigerated transport (by road and/or rail).

Finally, arcs link the cold stores to either the Fresh Produce Terminals (FPT), the South African Fruit Terminals (SAFT), the container terminals (Cape Town - CTCT, Durban - DCT and Port Elizabeth - PECT) or to cold stores near the ports, via decision nodes representing refrigerated transport in break-bulk and/or containers. Decision nodes are also present to accommodate decisions as to whether fruit should be packed into containers at the super-cold stores and transported by vehicles with generators attached to them (in order to provide electricity for the cooling of the container), or whether fruit should be transported in break-bulk by refrigerated vehicles.

Fruit is either refrigerated in a cold store until an export vessel is ready for loading, when it is packed into a container at the cold store for refrigerated transportation to a container terminal or transported directly to the wharf at a terminal, from where it is loaded onto a vessel. Fruit in containers is usually delivered to a container stack (only on rare occasions directly to a vessel) at a container terminal, where it is stored until the container is to be loaded onto the container vessel for export. The ports are the most complex part of the infrastructure network - the section of the network representing the port of Cape Town is shown in some detail in Figure 5.

In order to find the maximum permissible flow through a network, it is standard practice to modify the network first. All nodes with positive supply values (sources of flow) have to be joined to a single virtual source node, and all flow destinations with negative supply values have to be joined to a single virtual sink node. There are two main methodologies on which network maximum flow algorithms in the literature are based, namely

- those in which intermediate solutions remain feasible and satisfy a conservation of flow law (except at the source and sink nodes), by adjusting the flow incrementally 


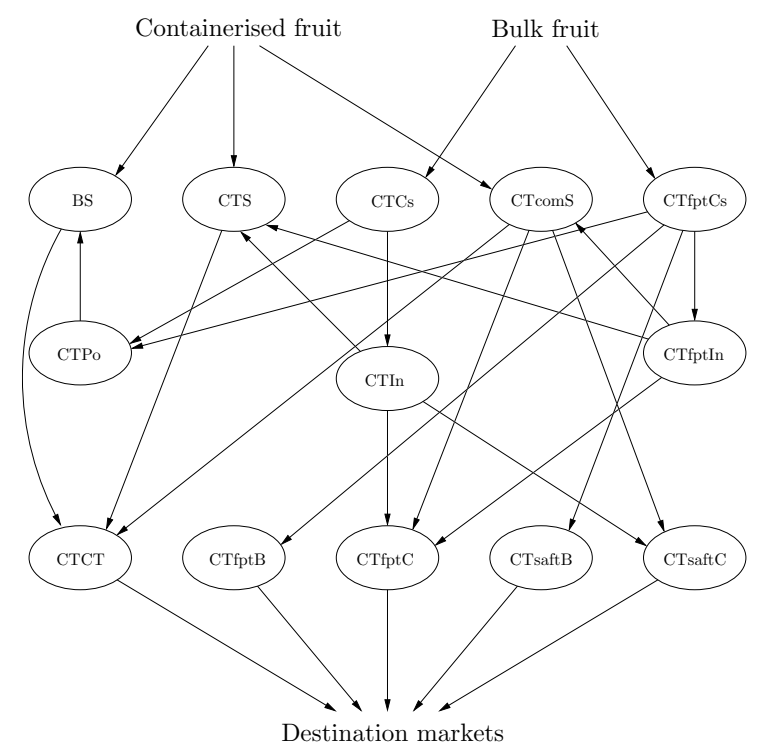

BS - Blue Store

CTS - Container stack

CTCs - Cold store near harbour

CTcomS - Multi-purpose container stack

CTfptCs - FPT cold store

CTPo - Porthole container packed near port

CTIn - Integral container packed near port

CTfptIn - Integral container packed at FPT

CTCT - Container terminal

CTfptB - Bulk loading at FPT

CTfptC - Container loading at FPT

CTsaftB - Bulk loading at SAFT

CTsaftC - Container loading at SAFT

Figure 5: Simplification of a part of the export infrastructure network representing the possible flow of fruit through the infrastructure components in the port of Cape Town. Note that the intake and discharge nodes for all the elements of this part of the network have been condensed into single nodes, for the sake of clarity.

along paths that start at the source and end at the sink, until optimality is achieved (known as augmenting path algorithms), and

- those that "push" more flow through the network than is actually allowed, so that some flow is either "sent back" to the source or "sent forward" to the sink, incrementally reducing the flow where necessary until optimality is achieved (known as preflow-push algorithms).

Ahuja, et al. $[1,2]$ discuss eleven algorithms (in three groups) that may be used to solve the maximum flow problem. The first is a collection of algorithms based on the Labelling algorithm (which has an $O(n m U)$ worst case running time, where $n$ is the number of nodes in the network, $m$ is the number of arcs and $U$ is the maximum value of the flow upper bounds on the arcs). The fastest of these algorithms is the Successive shortest path algorithm which has a running time of $O\left(n^{2} m\right)$. The second group of algorithms encompasses those based on the Generic preflow-push algorithm (which has a worst case running time of $O\left(n^{2} m\right)$ ). This group includes the fastest algorithm tested by Ahuja, et al. [2], namely the Highest-label preflow-push algorithm with a worst-case running time of $O\left(n^{2} \sqrt{m}\right)$. The third group is a sub-group of the preflow-push algorithms and consists of algorithms based on the Excess scaling algorithm (which has a worst case running time of $\left.O\left(n m+n^{2} \log U\right)\right)$, the fastest being the Stack scaling excess flow algorithm with a running time of $O\left(n m+n^{2} \sqrt{\log U}\right)$. After careful consideration of these alternatives as well as the properties and size of the export infrastructure network model, it was decided that the Successive shortest path algorithm [9] was well suited for our purposes and we implemented this algorithm in Fortran. 


\subsection{Multi-commodity mathematical programming model}

A major shortcoming of the graph theoretic approach in $\S 2.1$ is that it is cumbersome to incorporate multi-commodity flow specifications. As fruit types have different temperature control protocols set by the PPECB, and because packing rates are typically fruit type dependent, the different fruit types should ideally be considered as separate commodities (see Table 1 for a list of fruit groups), for which flow constraints through the export infrastructure differ.

\begin{tabular}{ll}
\hline Fruit group & Included fruit types \\
\hline Grapes & Table grapes \\
Stone & Apricots, nectarines, peaches, plums \\
Pome & Apples, pears \\
Hard citrus & Oranges, grapefruit, lemons, limes \\
Soft citrus & Mandarins \\
Avocados & Avocados \\
Mangos & Mangos \\
Litchis & Litchis \\
\hline
\end{tabular}

Table 1: The different fruit groups determined during the data gathering process by studying the cold chain protocols set by the PPECB. Note that fruits belonging to the soft citrus group are often called easy-peelers.

A multi-commodity approach is also important because only specific fruit types may flow through certain portions of the infrastructure. For example, only hard citrus fruit may be transported at ambient temperatures - arcs representing ambient transportation modes should therefore be inaccessible to other fruit types.

\subsubsection{Flow constraints}

Role-players in the export supply chain are often contractually obligated to send some minimum volume of fruit to particular pack houses, cold stores or terminals, or to utilise certain modes of transport. In such cases network arcs leading to and/or from the specific nodes in question may be assigned lower bounds on the volume of fruit that should be sent via such nodes. These considerations lead to a set of constraints of the form

$$
0 \leq L_{i j}^{k} \leq x_{i j}^{k}, \quad(i, j) \in \mathcal{A}, k \in \mathcal{K},
$$

stating that the flow volume $x_{i j}^{k}$ of commodity $k \in \mathcal{K}$ along the arc $(i, j)$ in the set $\mathcal{A}$ of all network arcs, must be at least some lower bound value, $L_{i j}^{k} \geq 0$. On the other hand, maximum flow constraints may represent capacities of pack houses, cold stores or terminals, such as the maximum rate at which a pack house may pack, the maximum rate at which cranes at the terminals are able to load fruit onto a vessel, etc. As this kind of limitation usually occurs at network nodes, a constraint set of the form

$$
\sum_{(i, j) \in \mathcal{N}(j)} x_{i j}^{k} \leq U_{j}^{k}, \quad j \in \mathcal{V}, k \in \mathcal{K}
$$

was incorporated into the model, where $\mathcal{V}$ denotes the vertex set of the network and where $\mathcal{N}(j)$ denotes the set of arcs directed from network facilities towards $j \in \mathcal{V}$. Here 
$U_{j}^{k}$ denotes an upper bound on the volume of commodity $k$ that may pass through node $j$ per time unit.

\subsubsection{Ambient citrus restriction}

Only a certain percentage of the hard citrus on an export vessel may be transported at ambient temperature. This percentage is determined by the PPECB, taking into account the shape of the hold and the cooling system used by the vessel. On average, $31 \%$ of all fruit has been exported at ambient temperatures in the past [17]. Thus the constraint

$$
\sum_{(i, j) \in \mathcal{U}} x_{i j}^{C} \leq \chi\left(\sum_{(i, j) \in \mathcal{R}} \sum_{k \in \mathcal{K}} x_{i j}^{k}+\sum_{(i, j) \in \mathcal{U}} x_{i j}^{C}\right), \quad 0 \leq \chi \leq 1
$$

was imposed, where $\mathcal{U}$ is the set of all arcs representing routes incident to a terminal $j$ to which ambient hard citrus is transported. Here $\mathcal{R}$ is the set of all arcs representing routes by which refrigerated fruit may be transported to a terminal $j, \chi$ is the fraction of hard citrus that may be loaded onto a vessel at ambient temperature (typically taken as 0.31) and $x_{i j}^{C}$ denotes the volume of hard citrus transported from facility $i$ which is adjacent to terminal $j$ in the network. This constraint may be simplified to

$$
\sum_{(i, j) \in \mathcal{R}} \sum_{k \in \mathcal{K}} x_{i j}^{k} \geq\left(\frac{1}{\chi}-1\right) \sum_{(i, j) \in \mathcal{U}} x_{i j}^{C}, \quad 0 \leq \chi \leq 1 .
$$

\subsubsection{Conservation of flow constraints}

All fruit flowing through the export infrastructure from production regions should be accounted for in the model. Therefore a conservation of flow law is included: All fruit flow into node $i$, namely $\sum_{h} x_{h i}^{k}$, subtracted from all fruit flow out of the same node, namely $\sum_{j} x_{i j}^{k}$, should equal the supply of that node, for each commodity $k$. Thus all nodes representing production regions will have a positive supply/net flow (since there is no flow into such nodes) and all nodes representing foreign destination markets will have negative supply values (as there is no flow out of such nodes). If one ignores all fruit rejected by the PPECB, packed for the local market or processed in South Africa (i.e., if one considers only export fruit), then all intermediate nodes should have zero supply values, which gives rise to a constraint set of the form

$$
\sum_{(i, j) \in \mathcal{A}} x_{i j}^{k}-\sum_{(h, i) \in \mathcal{A}} x_{h i}^{k}=b_{i}^{k}, \quad i \in \mathcal{V}, k \in \mathcal{K}
$$

where $b_{i}^{k}$ denotes the net supply volume of fruit commodity $k$ at node $i$. 


\subsubsection{The final model}

The objective in the multi-commodity mathematical programming model is therefore to

$$
\left.\begin{array}{rlrl}
\text { maximize } z & =\sum_{k} \sum_{(i, j) \in \mathcal{T}} x_{i j}^{k} & & \\
\text { subject to } x_{i j}^{k} & \geq L_{i j}^{k} \geq 0, & & (i, j) \in \mathcal{A}, k \in \mathcal{K}, \\
\sum_{(i, j) \in \mathcal{N}(j)} x_{i j}^{k} & \leq U_{j}^{k}, & & j \in \mathcal{V}, k \in \mathcal{K}, \\
\sum_{(i, j) \in \mathcal{R}} \sum_{k \in \mathcal{K}} x_{i j}^{k} & \geq\left(\frac{1}{\chi}-1\right) \sum_{(i, j) \in \mathcal{U}} x_{i j}^{C}, & & 0 \leq \chi \leq 1, \\
\sum_{(i, j) \in \mathcal{A}} x_{i j}^{k}-\sum_{(h, i) \in \mathcal{A}} x_{h i}^{k} & =b_{i}^{k}, & & j \in \mathcal{V}, k \in \mathcal{K},
\end{array}\right\}
$$

where $\mathcal{T}$ is the set of arcs that are adjacent to nodes representing loading areas at the container, FPT or SAFT terminals, where $\mathcal{A}$ is the set of all arcs and where $\mathcal{K}$ is the set of all commodity types.

The variables representing the flow $\left(x_{i j}^{k}\right)$ are decision variables which are to be found when solving the model - the solution will thus indicate the volume of fruit that should flow along the link between nodes $i$ and $j$ in an optimal solution. The values represented by $L_{i j}^{k}, U_{i j}^{k}, b_{i}^{k}$ and $\chi$ are parameters that are to be found or estimated before the model may be solved. A standard branch-and-bound procedure [22] may be used to solve this model.

\section{Collection of data}

A significant body of data was required to solve the models in $\S 2$ and since the fruit industry is composed of many role-players (such as pack house, cold store and terminal managers, transport operators, etc.), the gathering of these data has been a mammoth task. The data available suggested that a suitable model time step would be one week (i.e., working with weekly flow rates in the model).

\subsection{Fruit production and export volumes}

The fruit production figures used for subtropical fruits (avocados, mangos and litchis) derive from various sources. The South African Mango Growers' Association (SAMGA) [7] provided information on 2003 mango and litchi exports that had been provided to them by the PPECB. This information included the volume of export fruit that was inspected and rejected in each production region and was used in conjunction with weekly shipping data to estimate the export volumes of litchis and mangos per region per week. The South African Avocado Growers' Association (SAAGA) [18] was also able to provide detailed statistics that listed the export of each cultivar of avocado per region per week.

For deciduous fruit, tree census data [6] were used to estimate the percentage production per region of the total production. These estimates were derived with the help of DB Louw [12]. The total fruit production volume per week was used to estimate the weekly production percentages, so that when the two percentages were multiplied together, a per region per week production estimate could be made if the total production for a season was known. In cases where two years of weekly export data were available (2002-2003), the estimated production per week was averaged. 


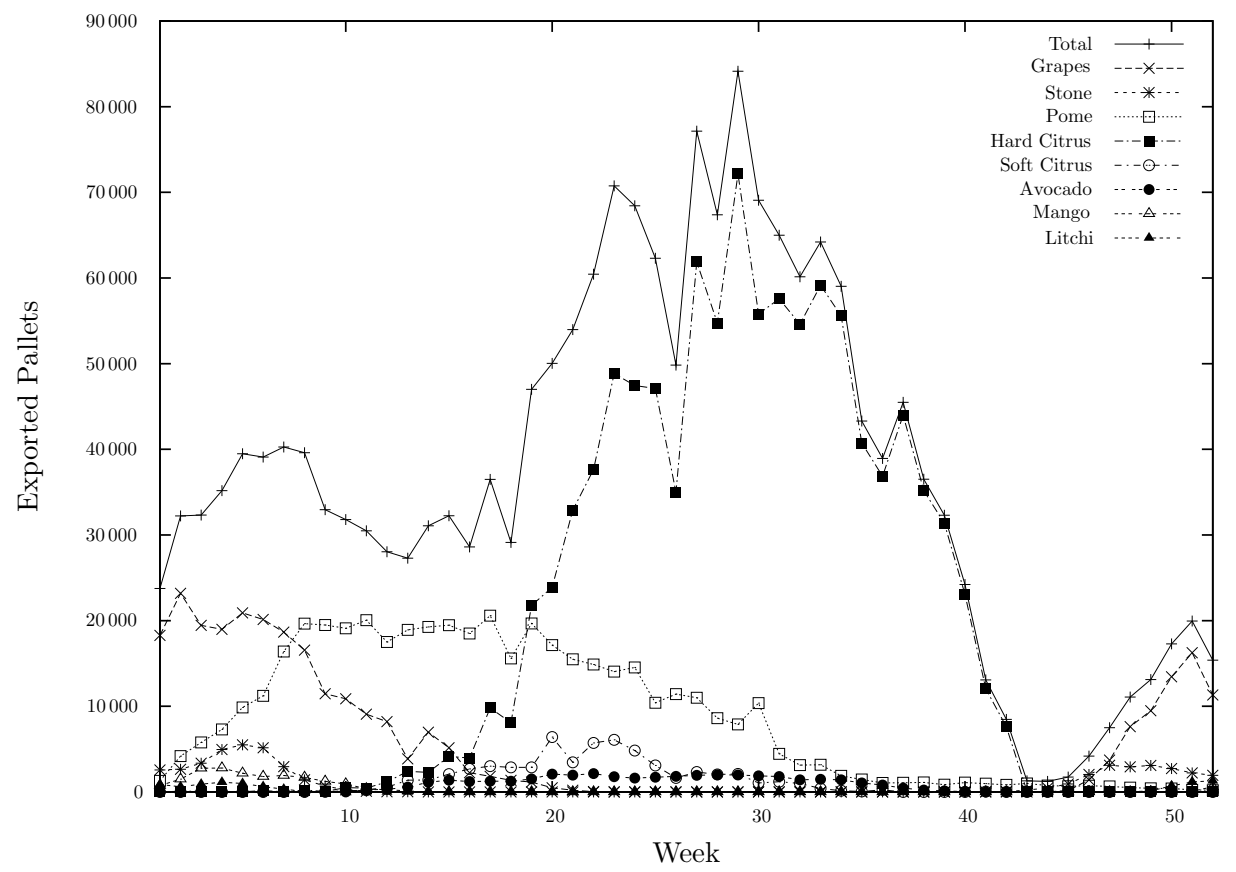

Figure 6: Pallets of fresh fruit exported per week from South Africa during 2003. Note that the deciduous fruit volumes are estimates, that the end-of-year volumes for mangos and litchis are approximations based on 2002 volumes, and that the volumes for citrus and avocados are accurate as reported by the CSA and SAAGA, respectively.

For citrus fruit, very detailed weekly shipping data were available from Citrus South Africa (CSA) [21]. This included the volume of fruit that was shipped, the production region from where the fruit originated, and the target market, which allowed for the compilation of a very accurate data set.

The resulting 2003 export volumes of the eight fruit groups listed in Table 1 are shown graphically in Figure 6.

\subsection{Pack house and cold store capacities}

The models in $\S 2$ require the maximum number of fruit pallets that may be packed in pack houses and that may be cooled to the correct temperatures by cold stores on a weekly basis. To gather these data, the PPECB was approached for a list of all pack houses and cold stores country wide. According to their information there are 850 pack houses and 315 cold stores throughout South Africa. All pack houses in which only grapes are packed were disregarded (since each grape farmer has his/her own pack house, as mentioned in $\S 2)$. This significantly decreased the number of role-players that had to be contacted in order to gather capacity data.

Managers of the remaining pack houses and of all cold stores were sent short questionnaires by email or fax in order to collect capacity and other data from them. Reminders were sent out as appropriate and this was followed up by telephonically contacting the managers of 
those facilities that did not respond. The total pack house and cold store capacities per week are listed for each region in Table 2, and the approximate locations of these regions may be found in Figure 2 .

\subsection{Terminal capacities}

The capacities of the four export terminals were estimated using a spreadsheet model designed by Van Dyk and Maspero [20]. The estimation procedure is explained in this section for the cases of the FPT, SAFT and CTCT terminals in the port of Cape Town. The terminal capacities for the other ports were estimated in the same way, but the details are omitted for the sake of brevity (however, details of these omitted calculations may be found in [16]). A summary of the resulting terminal capacities is shown in Table 3.

\subsubsection{Loading capacity at FPT Cape Town}

FPT in Cape Town has 3 berths, each guaranteeing a loading rate of 2000 fruit pallets per day (if required). Each day consists of two eight hour shifts. Assuming that the average vessel size is 4500 pallets under deck, it therefore takes approximately $2 \frac{1}{4}$ days (or 2 days and 4 hours) to load a vessel. Approximately 80 containers then have to be loaded onto the deck on average, but only after an average of 100 empty containers have been offloaded. Assuming that between 8 and 10 containers can be loaded on deck per crane hour and that two cranes are used, it takes approximately 9 hours to offload and load all containers on deck. The total time it takes to fill an entire vessel is therefore approximately 2 days and 13 hours. Allowing for berthing and deberthing, taking into account that only 16 hours are worked per day gives a total of 3 days to berth, load and deberth a vessel. Thus, the maximum number of fruit pallets that can be loaded at FPT Cape Town is approximately 7 days/week $\div 3$ days/vessel/berth $\times 3$ berths $=7$ vessels/week

Now that the number of vessels that may be serviced per week is known, it is possible to estimate the number of pallets that may be loaded onto vessels in containers and in break-bulk. The maximum break-bulk loading rate may be estimated as 7 vessels/week $\times 4500$ pallets $/$ vessel $=31500$ pallets $/$ week. Similarly, the maximum container loading rate is 7 vessels/week $\times 20$ pallets/container $\times 80$ containers/vessel $=11200$ pallets $/$ week. These figures account for the first two entries in Table 3.

\subsubsection{Loading capacity at SAFT Cape Town}

There is only a single berth at SAFT in Cape Town. They guarantee a loading rate of 2200 pallets per day (comprising two eight hour shifts); hence it takes approximately 2 days and an hour to load a vessel with a capacity of 4500 pallets of fruit under deck. Assuming it takes 5 hours for two cranes to offload an average of 100 empty containers and 4 hours to load the containers on deck, and 5 hours to deberth the vessel and berth the next one, it takes approximately 2 days 15 hours, or more realistically 3 days, to turn a single vessel around. Hence, SAFT is able to load 7 days/week $\div 3$ days/vessel $=2.33$ vessels/week. Therefore SAFT is able to load a maximum of 2.33 vessels/week $\times 4500$ pallets/vessel $=10500$ pallets/week in break-bulk and a further 2.33 vessels/week $\times 20$ 


\begin{tabular}{|c|c|c|c|}
\hline Region & $\begin{array}{c}\text { Region } \\
\text { Code }\end{array}$ & $\begin{array}{r}\text { Pack house } \\
\text { Capacity }\end{array}$ & $\begin{array}{r}\text { Cold Store } \\
\text { Capacity }\end{array}$ \\
\hline Levubu & Le & 11510 & 1006 \\
\hline Tzaneen & $\mathrm{Tz}$ & 17258 & 2886 \\
\hline Hoedspruit & Ho & 4075 & 2139 \\
\hline Kiepersol & $\mathrm{Ki}$ & 1924 & 2644 \\
\hline Nelspruit & $\mathrm{Ne}$ & 4405 & 891 \\
\hline Malelane & $\mathrm{Ma}$ & 7151 & 1776 \\
\hline Potgietersrus & Po & 2365 & - \\
\hline Ellisras & $\mathrm{El}$ & - & 770 \\
\hline Marble Hall & $\mathrm{Mh}$ & 9365 & 1670 \\
\hline Ermelo & Er & 400 & - \\
\hline Pongola & $\mathrm{Pg}$ & 1454 & - \\
\hline Nkwalini & $\mathrm{Nk}$ & 7445 & 25 \\
\hline KwaZulu-Natal & $\mathrm{Kz}$ & 1294 & 1190 \\
\hline Eastern Cape Midlands & $\mathrm{Ea}$ & 688 & 460 \\
\hline Sundays River Valley & $\mathrm{Su}$ & 10351 & 3000 \\
\hline Patensie & $\mathrm{Pa}$ & 6559 & 2338 \\
\hline Langkloof & $\mathrm{La}$ & 11748 & 9187 \\
\hline Karoo & $\mathrm{Ka}$ & 366 & - \\
\hline Klein Karoo & $\mathrm{Kl}$ & 2571 & 483 \\
\hline GEVV & $\mathrm{Ge}$ & 19436 & 28299 \\
\hline Boland & Bo & 5902 & 6527 \\
\hline Ceres & $\mathrm{Ce}$ & 15451 & 9158 \\
\hline De Doorns & $\mathrm{De}$ & - & 13904 \\
\hline Piketberg & $\mathrm{Pi}$ & 2428 & 4550 \\
\hline Citrusdal & $\mathrm{Ci}$ & 6428 & 300 \\
\hline Orange River & Or & 135 & 80026 \\
\hline Hartswater & $\mathrm{Ha}$ & 1557 & 248 \\
\hline Johannesburg & Jo & 310 & 2296 \\
\hline Rustenburg & $\mathrm{Ru}$ & 58 & 36 \\
\hline Free State & Fs & 783 & 1180 \\
\hline Middelburg & Mi & 9800 & - \\
\hline Total & & 163217 & 176919 \\
\hline
\end{tabular}

Table 2: Capacities of pack houses and cold stores (measured in pallets processed per week) in each of the 31 the fruit production regions of South Africa. (GEVV represents the area around the Western Cape towns of Grabouw, Elgin, Villiersdorp and Vyeboom.) Note that the cold stores listed for the Johannesburg region include those at the airport. The cold stores in or near ports are omitted. 


\begin{tabular}{lrrrrrr}
\hline \multirow{2}{*}{ Port } & \multicolumn{2}{c}{ FPT } & \multicolumn{2}{c}{ SAFT } & \multicolumn{2}{c}{ Cont Term } \\
\cline { 2 - 7 } & Bulk & Cont & Bulk & Cont & Stack & Loading \\
\hline Cape Town & 31500 & 11200 & 10500 & 3733 & 31390 & 45840 \\
Durban & 45320 & 7769 & 13140 & 2253 & 12720 & 103940 \\
Port Elizabeth & 22615 & 3877 & - & - & 11480 & 50540 \\
Maputo & 22615 & 3877 & - & - & - & - \\
\hline
\end{tabular}

Table 3: Capacities (in pallets per week) of the four terminals considered in the models of $\S 2$. Legend: Bulk $=$ Break-bulk, Cont $=$ Containerised, Stack $=$ Container stack and Loading $=$ Containers loaded onto vessels.

pallets/container $\times 80$ containers/vessel $=3733$ pallets/week in containers on the decks of conventional vessels. These figures account for the third and fourth entries in Table 3.

\subsubsection{Loading capacity at CTCT}

The CTCT stacks (which have 995 plug-in points) close twice weekly; hence the reefer stack may be used twice a week. On average, 319 non-fruit containers pass through the stack during a week, leaving $995 \times 2-319=1599$ plug points for export fruit integral containers. According to data obtained from South African Port Operations in Cape Town, $3.7 \%$ of all integral containers passing through the stack are $6 \mathrm{~m}$ containers and the remaining $96.3 \%$ are $12 \mathrm{~m}$ integral containers. This means that $(1599 \times 3.7 \% \times 10)$ $+(1599 \times 96.3 \% \times 20) \approx 590+30800=31390$ pallets of fruit may potentially pass through the stack per week. This figure accounts for the fifth entry in Table 3.

Finally, there are 6 gantry cranes at CTCT that each achieves 17 moves per crane hour during peak weeks (but usually one crane is down for maintenance, i.e. 5 cranes would operate at any one time). A total of 3 berths may accommodate container vessels, thus $17 \times$ $6 / 3=34$ containers may either be loaded or offloaded per hour per berth. However, due to practical reasons each eight hour shift usually only consists of 7.5 effective working hours. For vessels with an average call size of 446 containers, it therefore takes approximately $446 /(34 \times 7.5 / 8)=14$ hours to complete the offloading and loading. Assuming it takes 5 hours to deberth the vessel and berth the next, the average turn-around time for a vessel is estimated to be 19 hours. The container terminal can therefore handle $24 \times 7 / 19=8.84$ vessels per berth per week, or 26.5 per week in total. This means that $26.5 \times 446=11831$ container moves may be performed per week. Since approximately the same number of containers are offloaded as are loaded in Cape Town, and an average of 2999 non-fruit containers are loaded onto vessels per week, 11831/2 - 2999 = 2916 container export moves per week remain that may possibly be utilised by fruit integral containers. Of all fruit containers that are loaded onto vessels, $42.8 \%$ are $6 \mathrm{~m}$ reefer containers (either porthole or integral) and the remaining $57.2 \%$ are $12 \mathrm{~m}$ integrals. The additional containers that are not loaded via the stack are either porthole containers, or direct shipments. Thus $(2916 \times 42.8 \% \times 10)+(2916 \times 57.2 \% \times 20) \approx 12480+33360=45840$ pallets of fruit may potentially be loaded onto vessels per week. This figure accounts for the sixth entry in Table 3. 


\subsection{Road and rail capacities}

Van Dyk and Maspero [20] discuss South African road capacities in the context of fresh fruit transportation in their key findings. According to their information (acquired during Phase 4 of the Fruitlog study) there currently do not seem to be any significant capacity constraints on road usage. The reported problems encountered by vehicles near ports during peak hour congestion or at the check points of terminals were not taken into account - this omission may be justified, as will be explained in the conclusion. Similarly the capacities of rail transportation do not seem to limit fruit export from a practical point of view. It was therefore decided to place such high flow volume upper bounds on network arcs representing road or rail transport infrastructure segments, that flow along these links would be unconstrained for all practical purposes.

\section{Model implementation scenarios}

The models in $\S 2$ were solved for a number of test scenarios, but we report the results for two of these test scenarios here. One of these scenarios was chosen to coincide with a week in which peak volumes of fruit are exported during summer and the second scenario was chosen to coincide with winter peak export volumes. The 2003 weekly fruit export volumes are shown in Figure 6 and the 2003 export volumes during the peak weeks of each season are summarised in Table 4.

\begin{tabular}{lrr}
\hline Fruit type & Week 7 & Week 29 \\
\hline Grapes & 18653 & 0 \\
Stone & 4398 & 0 \\
Pome & 15718 & 8581 \\
Hard citrus & 23 & 70043 \\
Soft citrus & 0 & 2109 \\
Avocados & 0 & 1989 \\
Mangos & 1120 & 0 \\
Litchis & 327 & 0 \\
\hline Total & 40239 & 82722 \\
\hline
\end{tabular}

Table 4: Fruit export volumes (measured in pallets) during week 7 of 2003 (summer peak) and week 29 of 2003 (winter peak).

\subsection{Summer peak scenario}

The fruit groups exported in summer generally have a very short shelf life (especially subtropical and stone fruit) - thus exporters attempt to transport their fruit to foreign markets as quickly as possible. This is achieved by transporting fruit over land to Cape Town, as it is the port closest to the major summer markets (such as Europe and the Americas) and thus the shipping time is shortest. Shipping lines dealing with uncontainerised fruit also tend not to service the other three major Southern African ports during this time. Small volumes of containerised fruit destined for the Far East may nevertheless be exported via the other ports during this period. However, the port of Cape Town was 
taken as the only exit point for fruit export in the summer peak scenario. The error induced by this assumption is expected to be negligible.

The single-commodity graph theoretic model consists of 670 nodes and 2890 arcs for this scenario and predicts a maximum volume of fruit export from Cape Town during the summer peak week of 100523 pallets. The multi-commodity mathematical programming model predicts the same maximum volume of fruit flow per week and consists of 906 constraints and 2542 decision variables.

\begin{tabular}{lrrrrrrr}
\hline Fruit & \multicolumn{5}{c}{ Possible Export Volume } & 2003 \\
\cline { 2 - 5 } Type & FPT (B) & SAFT (B) & FPT (C) & SAFT (C) & Cont. Term. & Total & Volumes \\
\hline Grapes & 2099 & 0 & 0 & 162 & 38050 & 40311 & 18653 \\
Stone & 0 & 8015 & 0 & 0 & 0 & 8015 & 4398 \\
Pome & 8186 & 0 & 7143 & 631 & 431 & 16391 & 15718 \\
Citrus & 19757 & 2485 & 4057 & 1128 & 5000 & 32427 & 23 \\
Mango & $\mathrm{n} / \mathrm{a}$ & $\mathrm{n} / \mathrm{a}$ & 0 & 1812 & 109 & 1921 & 1120 \\
Litchi & 1458 & 0 & 0 & 0 & 0 & 1458 & 327 \\
\hline Total & 31500 & 10500 & 11200 & 3733 & 43590 & 100523 & 40239 \\
\hline
\end{tabular}

Table 5: The maximum possible flow of fruit (in pallets per week) through the South African fruit export infrastructure in summer, assuming that the port of Cape Town is the only point of exit from the export infrastructure. Legend: $B=$ Break-bulk, $C=$ Containerised, $n / a=n o t$ allowed, Cont. Term. = Container terminal.

The volume of fruit exported from each production region in the maximum flow solution is not interesting, because all bottlenecks occur at the port in this scenario - no bottlenecks occur at a regional pack house or cold store level. This means that all exported fruit may be drawn from a select few production regions by the Successive shortest path algorithm. One could incorporate additional constraints in the model so as to force each region to export at least the volume of fruit that was exported during 2003. However, this does not increase the relevance of the model solution as all fruit volumes flow to a single port, and to the models the origin of fruit is of no consequence, since no transportation costs were incorporated into the models. Because the port infrastructures are the bottleneck, and since their capacities are not dependant on fruit type differentiation, the two models produce the same maximum flow volume for this scenario. A breakdown of the results (for each fruit group) for the multi-commodity model maximum flow solution is shown in Table 5 .

To determine whether a binding constraint (such as the capacity of a cold store or stack) is, in fact, a bottleneck, the shadow price of the constraint had to be an integer (depending on the weighting given to the flow). This means that the value of the model objective function would increase by one unit for every unit by which the binding constraint is relaxed. The shadow price was found on the sensitivity report made available by the XPRESS-MP mixed-integer linear programming solver [4] that was used to solve the mathematical programming model (this solver was accessed via the NEOS Server for Optimisation [3]). One finds that some infrastructure components on pack house and cold store levels are used to capacity. Although the binding constraints seem to be bottlenecks at a first glance, they are, in fact, not. This is often the case in optimisation problems where there are 
multiple decision options and the coefficients of the variables in the objective function are all the same, leading to non-uniqueness of optimal solutions. Thus, if one increases the capacity of an element in the infrastructure which is not a bottleneck, but which is associated with a binding constraint (such as the container stack in Cape Town), flow from another element of the infrastructure may be diverted in that direction. This means the flow through the first part of the infrastructure will increase, but the overall flow of the system remains the same as the flow through another part will simply decrease.

The maximum break-bulk loading rates at both the FPT and SAFT terminals are reached in the maximum flow solution, as are the maximum container loading rates at those terminals. Both the Cape Town stack and the combi-stack at the multi-purpose terminal are fully utilised so as to achieve the maximum flow, and the Blue Store turnover is at its capacity of 500 containers a week.

The restriction on the volume of ambient citrus allowed into vessels at the port is not a limiting factor in this scenario, nor is the loading rate of containers from stacks onto vessels. The gantry cranes are able to load an additional $45840-43590=2250$ pallets a week over and above what is required for the maximum flow solution. Assuming that the $6 \mathrm{~m} / 12 \mathrm{~m}$ ratio remains constant for any additional containers of fruit, an additional 82 twelve metre integral containers may be loaded onto container vessels (assuming there are enough container vessels), along with 61 additional six metre containers.

After observing the maximum flow solution described above, it was decided to assign additional objective function weights to each fruit group individually so as to ascertain where the first infrastructure bottleneck would occur if the production volumes of that fruit type were to increase dramatically, and if those of the other fruit groups were at the 2003 export levels. More specifically, the objective was thus to

$$
\operatorname{maximise} \sum_{\mathcal{K} \backslash\{F\}} \sum_{(i, j) \in \mathcal{T}} x_{i j}^{k}+2 \sum_{(i, j) \in \mathcal{T}} x_{i j}^{F}
$$

where $F$ represents one of the six summer fruit groups, where $\mathcal{K}$ is the set of all summer fruit groups, and where $\mathcal{T}$ is the set of arcs representing the loading capacities of vessels at each of the terminals (as shown in Figure 4 and discussed in $\S 2.1$ ). The sensitivity analysis results of the six resulting revised optimal solutions are summarised in Table 6 .

\subsection{Winter peak scenario}

The second scenario concerns the winter peak during week 29 (in 2003). In this week, only pome, hard citrus, soft citrus and avocados were exported during 2003. The singlecommodity graph theoretic model consists of 607 nodes and 2883 arcs for this scenario and predicts a maximum volume of fruit export from all ports during the winter peak week of 151346 pallets. However, the multi-commodity mathematical programming model predicts a maximum volume of fruit flow per week of 150004 pallets, and consists of 850 constraints and 4069 decision variables. The difference between the solutions of the two models is due to bottlenecks occurring at a pack house and cold store level, where fruit group differentiation is important with regards to capacities, as opposed to the bottlenecks in the summer peak scenario occurring at terminal level, where fruit group differentiation is 


\begin{tabular}{lrrl}
\hline $\begin{array}{l}\text { Fruit type } \\
\text { additionally } \\
\text { weighted }\end{array}$ & $\begin{array}{r}\text { New max } \\
\text { export } \\
\text { volume }\end{array}$ & $\begin{array}{r}\text { \% of capacity } \\
\text { reached during } \\
\text { summer 2003 }\end{array}$ & Bottlenecks \\
\hline Grapes & 78901 & $29 \%$ & Port of Cape Town \\
\hline Stone & 25014 & $52 \%$ & $\begin{array}{l}\text { Pack houses in the Patensie, Langkloof, Klein Karoo, } \\
\text { GEVV, Boland, Ceres, Piketberg, Citrusdal, Johan- } \\
\text { nesburg and Rustenburg regions. Cold stores in the } \\
\text { Klein Karoo and Hartswater regions. }\end{array}$ \\
\hline Pome & 39524 & $\begin{array}{l}\text { and Citrusdal regions. Cold stores in the Langkloof, } \\
\text { Boland, Ceres and Free State regions. }\end{array}$ \\
\hline Citrus & $0.3 \%$ & $\begin{array}{l}\text { A large increase in citrus production during the sum- } \\
\text { mer peak is considered highly unlikely and therefore } \\
\text { an unrealistic scenario. }\end{array}$ \\
\hline Mango & $30 \%$ & $\begin{array}{l}\text { Pack houses in the Hoedspruit and Kiepersol regions. } \\
\text { Cold stores in the Levubu, Tzaneen, Nelspruit and } \\
\text { Malelane regions. }\end{array}$ \\
\hline Litchi & $13 \%$ & $\begin{array}{l}\text { Pack houses in the Levubu, Tzaneen, Kiepersol and } \\
\text { Malelane regions. Cold stores in the Nelspruit region. }\end{array}$ \\
\hline
\end{tabular}

Table 6: Sensitivity analysis of the weekly maximum export volume (measured in number of pallets) of each of the six summer fruit types (if all other fruit export volumes were to remain at 2003 export levels), with only the port of Cape Town as infrastructure exit point.

not important. A breakdown of the results (for each fruit group) for the multi-commodity model maximum flow solution is shown in Table 7 .

\begin{tabular}{lrrr}
\hline \multirow{3}{*}{ Fruit Group } & \multicolumn{3}{c}{ Flow (pallets) } \\
\cline { 2 - 4 } & $\begin{array}{r}\text { Maximum } \\
\text { flow (GT) }\end{array}$ & $\begin{array}{c}\text { Maximum } \\
\text { flow (MP) }\end{array}$ & $\begin{array}{r}2003 \\
\text { flow }\end{array}$ \\
\hline Pome & 39854 & 33680 & 8581 \\
Hard citrus & 104001 & 102351 & 70043 \\
Soft citrus & 3164 & 9546 & 2109 \\
Avocado & 4327 & 4427 & 1989 \\
\hline Total & 151346 & 150004 & 82722 \\
\hline
\end{tabular}

Table 7: The maximum export volume (for the graph theoretic (GT) and mathematical programming (MP) models) of each individual fruit group in the winter scenario, compared to the actual export volumes of fruit during the 2003 season.

Most of the pack houses would have to operate at full capacity to achieve the maximum flow solution in Table 7. In the Levubu region, pack houses for hard citrus, soft citrus and avocado are stretched to the limit to achieve a total export of 11250 pallets of fruit. In Tzaneen only the hard citrus pack houses are bottlenecks, while there is capacity for an additional 142 pallets a week of avocados. The total export from Tzaneen may be as much as 16425 pallets of fruit. In Hoedspruit (total export may be as high as 3181 pallets of hard citrus and soft citrus), Kiepersol (total export could be 1924 pallets of hard 
citrus and avocados), Nelspruit (total export potential of 4273 pallets of hard citrus and avocado), Malelane (could export up to 5960 pallets of hard citrus), Potgietersrus (2365 pallets of hard citrus at maximum), Marble Hall (9365 pallets of hard citrus and soft citrus), Pongola (1 454 pallets of hard citrus), Nkwalini (7 445 pallets of hard citrus, soft citrus and avocado), KwaZulu-Natal (1 294 pallets of hard citrus, soft citrus and avocado), Eastern Cape Midlands (688 pallets of hard citrus and soft citrus), Sundays River Valley (10351 pallets of hard citrus and soft citrus) and Patensie (6559 pallets of hard citrus and soft citrus) the pack houses are the bottlenecks. In the Langkloof region (potentially 10141 pallets of pome and hard citrus) only the hard citrus pack houses are bottlenecks, while in the GEVV region all pack houses are bottlenecks restricting the maximum export from this region to 18061 pallets of pome, hard citrus and soft citrus. In the Boland region the hard citrus pack houses are bottlenecks, but an export volume of 5884 pallets of pome, hard citrus and soft citrus may be achieved. In Ceres (potentially 11304 pallets of pome, hard citrus and soft citrus) only the restriction on hard citrus and soft citrus packing are bottlenecks, while in Piketberg (2 428 pallets of pome and hard citrus), Citrusdal (6428 pallets of pome, hard citrus and soft citrus), the Orange River (135 pallets of hard citrus and soft citrus), Hartswater (1070 pallets of hard citrus and soft citrus), Rustenburg (26 pallets of hard citrus) and Middelburg (9800 pallets of hard citrus per week) the pack houses are bottlenecks. In the Free State, packing facilities are not restrictive, allowing potentially 300 pallets of pome to be exported.

In Levubu, cold stores have a greater handling capacity per week than the pack houses, so they are not bottlenecks. In Tzaneen the cold stores handling avocados restrict the flow of avocados to 142 pallets and are thus potential bottlenecks, while the hard citrus cold stores in this region present no problem. In the Hoedspruit, Kiepersol, Nelspruit, Malelane, Marble Hall, Nkwalini, KwaZulu-Natal, Eastern Cape, Sundays River Valley and Patensie regions the cold stores do not restrict the volume of fruit that could potentially be exported; the pack houses are the bottlenecks there. In the Langkloof region the cold stores are the bottlenecks, allowing a total of 9187 pallets of fruit to pass though the facilities of the region, while the pack houses allow up to 11748 pallets of fruit to be packed (a difference of 2561 pallets). In the Klein Karoo and GEVV regions, cold stores are not restrictive to the flow of fruit. In the Boland region, the flow of pome fruit to the port of Cape Town is hindered by the capacity of the cold stores. Up to 4349 pallets of pome fruit may potentially be packed by the pack houses in the region, but infrastructure to cool only 1680 pallets exists (a 2669 pallets per week difference). In Ceres the cold stores restrict the flow of pome fruit to the port, while the De Doorns, Piketberg, Citrusdal, Orange River, Hartswater and Johannesburg regions have sufficient cold storage. The Free State lacks cold storage for its pome as only 300 pallets may be cooled to the required temperature in that region, while 783 pallets may be packed a week (a difference of 483 pallets per week).

A total of 47571 pallets of fruit should be exported during the winter peak week via containers to achieve maximum throughput, and the remaining 102433 pallets per week should be exported in break-bulk. 


\section{Conclusions and recommendations}

The results of the maximum flow models in $\S 2$ indicate that the infrastructure currently available to the South African fresh fruit export industry is more than adequate to accommodate the export levels of 2003, as well as those expected in the near future [8]. The largest volume of fruit that may be exported during the winter peak week is 150004 pallets of fruit, compared to the 82722 pallets that were actually exported during that busiest week of 2003 - this amounts to an approximate infrastructure utilisation of at most $55 \%$ in winter. Furthermore, even if only the port of Cape Town is considered as exit point during the peak summer week, the capacity of the port (100523 pallets per week) by far exceeds the 2003 export volume during that week (40239 pallets of fruit), which amounts to an approximate infrastructure utilisation of at most $40 \%$ in summer.

However, it is important to note that the figures arrived at for the loading rates at the port terminals (in $\S 3$ ), and the volumes of fruit that may be loaded directly onto export vessels (bypassing the cold stores) assume no interference or disruptions that would hinder the efficient functioning of the terminals. Some examples of such interference include:

- weather (high wind speeds and/or rain may slow down loading, or bring it to a complete halt),

- labour disruption (striking or unproductive workers may prevent 100\% efficiency),

- large volumes of traffic (preventing vehicles from arriving at the terminals when expected and hence disrupting the loading process which requires pallets or containers to be placed in a certain pattern or loaded in a certain order onto the vessel),

- incorrect documentation (resulting in fruit pallets not arriving at the berth on time),

- fruit that is not at the desired temperature as per the PPECB protocol [15],

- cold stores not being able to load fruit fast enough in the correct sequence (the large cold rooms in some cold stores may lead to difficulties in finding the exact pallets that should be transported to the port), and

- equipment breakdown (resulting in a direct loss of capacity).

Inevitably at least one of these disruptions may occur during the same week, which may reduce the modelled capacities significantly.

During visits to the port of Cape Town it became clear to the authors that vehicles would often arrive in batches, resulting in long queues (and hence a perceived port bottleneck), but once these vehicles had passed the various check points, there would typically follow periods during which the container terminal workers would be idle. This perceived bottleneck phenomenon is not fundamental to the infrastructure and its capacities, but are rather due to sub-optimal management of the existing infrastructure (a finding corroborated by Bekker, et al. [5]). The problem may be overcome by, for example, implementing a vehicle arrival schedule and penalty programme for latecomers so as to alleviate congestion. However, the design of such a schedule will have to be flexible enough to accommodate 
vehicles transporting cargo from destinations far away from the port - in such cases head winds, tyre punctures or any other traffic disturbances may result in vehicles easily not arriving on time and thus causing unnecessary congestion. Such an arrival schedule design process may profit from the creation of a truck-stop outside the port perimeter, where the drivers may check in their cargo and be assigned a delivery time dynamically.

For the winter peak week scenario $(\S 4.2)$ it was assumed that South African exporters generally prefer to send subtropical and deciduous fruit to the port of Cape Town. Fruit arriving by vehicle in Cape Town will have been picked later than fruit arriving in a vessel from Durban and should thus be fresher, allowing for a longer shelf-life, and the shorter sailing times to Europe and the Americas from Cape Town also make it an attractive port of departure. Due to the location of Durban on the east coast of South Africa, it is more appropriate for fruit exported to the Middle East, Far East, Japan and the Indian Ocean Islands, or for the export of fruit with a long shelf-life, such as citrus. The maximum flow solution of the winter peak week scenario may change significantly if port preferences are encapsulated in the model constraints.

There may also be other methods of making more efficient use of the current fruit export infrastructure. For example,

- if crane operators were to load a larger number of containers or pallets per hour, the capacity of the terminals may increase significantly. This is clearly shown by Van Dyk and Maspero [20], where the utilisation of the container terminal in particular drops significantly when fruit flow volumes remain constant and the number of containers loaded per hour is increased by three, and one extra crane is used. Further evidence of this may be found in the Fruit Logistics Scenario Model [19].

- increased efficiency of other infrastructure components may also increase the export volumes of fruit significantly. These possibilities are investigated in further studies $[5,11,13]$.

- diverting some fruit from congested ports (such as Durban during the winter peak weeks) to ports often not fully utilised may be investigated. For example, since Maputo is the closest port to the Mpumalanga, Limpopo and Swaziland production regions, the viability of diverting more citrus currently exported via Durban from these regions to Maputo, should be investigated. Such a diversion may be effective in view of the facts that extensive rehabilitation is taking place at the port of Maputo, that there is already a conventional fruit terminal in the port, and that there has been increased high-level effort to stimulate the Maputo corridor.

Finally, Van Dyk and Maspero [20] suggest a feasibility study for establishing a fruit terminal at the port of Lüderitz in Namibia. This has the potential of reducing the strain on the Cape Town terminals during the summer peak, when the Orange River and Namibian farmers export large volumes of table grapes. Building a fruit terminal in Lüderitz would also probably reduce the transportation costs of those farmers in the long term, but may itself be a costly short-term endeavour. 


\section{Acknowledgements}

Work in this paper was taken from the master's thesis [16] of the first author, whose studies ware funded by the Department of Labour's Scarce Skills Programme. The second author was funded by the National Research Foundation under Grant 2072815.

\section{References}

[1] Ahuja RK, Kodialam M, Mishra AK \& Orlin JB, 1997, Computational investigations of maximum flow algorithms, European Journal of Operations Research, 97, pp. 509-542.

[2] Ahuja RK, Magnanti TL \& Orlin JB, 1993, Network flows - Theory, algorithms and applications, Prentice-Hall, Englewood Cliffs (NJ).

[3] Argonne National Laboratory, 2005. NEOS server for optimisation, [Online], [Cited April 7th, 2005], Available from: http://www-neos.mcs.anl.gov

[4] Argonne National Laboratory, 2005. NEOS server: XpressMP, [Online], [Cited April 7th, 2005], Available from: http://www-neos.mcs.anl.gov/neos/ neos-5/Web/solvers/milp:XpressMP/MPS.html

[5] Bekker JF, Mostert M \& VAn Dyk FE, 2005, Simulation of fruit pallet movement in the port of Durban, ORiON, 21(1), pp. 63-75.

[6] Centre For International Agricultural Marketing And Development, 2003, Key Deciduous Fruit Statistics 2002.

[7] Crafford S, 2003, Personal communication, General Manager at the South African Mango Growers' Association, Contactable at: stephan@mango.co.za

[8] Du Bois A, 2005, Personal communication, Transport Manager at FPT, Cape Town, Contactable at: Albertyn_DuBois@fpt.co.za

[9] Evans JR \& Minieka E, 1992, Optimization algorithms for networks and graphs, $2^{\text {nd }}$ edition, Marcel Dekker, New York (NY).

[10] Kriegler J, 2003, Personal communication, President of the Hex Table Grapes Association, Contactable at jkriegler@mweb.co.za

[11] Kritzinger CC, 2003, Simulation model of FPT - Cape Town, (Unpublished) BEng (Industrial) final year project, University of Stellenbosch, Stellenbosch.

[12] Louw DB, 2003, Personal communication, owner of Optimal Agricultural Business Systems (OABS) based at the DFPT, Contactable at daan@dfpt.co.za

[13] Mostert M, 2003, The development of a computer simulation model of the Durban fresh produce terminal, (Unpublished) BEng (Industrial) final year project, University of Stellenbosch, Stellenbosch. 
[14] Murray X, 2004, Personal communication, Quality Control Manager for Monteith Trust Farms, Contactable at xavier@monteith.co.za

[15] Perishable Products Export Control Board, 2003, Carrying temperature regimes of fresh produce for sea export: Official PPECB instructions Rev22, [Online], [Cited April 10th, 2003], Available from http://www.ppecb.co.za/ Community/Procedures. asp

[16] Ortmann FG, 2005, Modelling the South African fruit export supply chain, MSc Thesis, University of Stellenbosch, Stellenbosch.

[17] Robinson R, 2004, Personal communication, Cold chain specialist at the PPECB, Contactable at RobbieR@ppecb.com

[18] South African Avocado Growers' Association, 2003, Personal communication, Contactable at saaga@saaga.co.za

[19] van Dyk FE, Havenga J, Ortmann FG, Hobbs I, Louw DB \& Louw NH, 2004, Fruit logistics scenario model: Port capacity and fruit export model, [Online], [Cited March 18th, 2005], Available from http://www.dfpt.co.za/ fruitlog/index.htm

[20] Van Dyk FE \& Maspero E, 2004, An analysis of the South African fruit logistics infrastructure, ORiON, 20(1), pp. 55-72.

[21] Vermank I, 2003, Personal communication, Information Manager at Citrus South Africa, Contactable at data@citrussa.co.za

[22] Winston WL, 1994, Operations research: Applications and algorithms, $3^{\text {rd }}$ edition, Duxbury Press, Belmont (CA). 
